\title{
Super-Eddington wind scenario for the progenitors of type la supernovae: binary population synthesis calculations
}

\author{
B. Wang ${ }^{1,2}$, X. Ma ${ }^{1,2}$, D.-D. Liu ${ }^{1,2}$, Z.-W. Liu ${ }^{3}$, C.-Y. Wu ${ }^{1,2}$, J.-J. Zhang ${ }^{1,2}$, and Z. Han ${ }^{1,2}$ \\ 1 Yunnan Observatories, Chinese Academy of Sciences, 650026 Kunming, PR China \\ e-mail: wangbo@ynao.ac.cn \\ ${ }^{2}$ Key Laboratory for the Structure and Evolution of Celestial Objects, Chinese Academy of Sciences, 650026 Kunming, PR China \\ 3 Argelander-Institut für Astronomie, Auf dem Hügel 71, 53121 Bonn, Germany
}

Received 7 November 2014 / Accepted 23 February 2015

ABSTRACT

Context. The super-Eddington wind scenario has been proposed as an alternative way for producing type Ia supernovae (SNe Ia). The super-Eddington wind can naturally prevent the carbon-oxygen white dwarfs (CO WDs) with high mass-accretion rates from becoming red-giant-like stars. Furthermore, it works in low-metallicity environments, which may explain SNe Ia observed at high redshifts.

Aims. In this article, we systematically investigated the most prominent single-degenerate WD+MS channel based on the superEddington wind scenario.

Methods. We combined the Eggleton stellar evolution code with a rapid binary population synthesis (BPS) approach to predict SN Ia birthrates for the WD+MS channel by adopting the super-Eddington wind scenario and detailed mass-accumulation efficiencies of $\mathrm{H}$-shell flashes on the WDs.

Results. Our BPS calculations found that the estimated SN Ia birthrates for the WD+MS channel are $\sim 0.009-0.315 \times 10^{-3} \mathrm{yr}^{-1}$ if we adopt the Eddington accretion rate as the critical accretion rate. These rates are much lower than those of the observations $(<10 \%$ of the observed SN Ia birthrates). This indicates that the WD+MS channel only contributes a small portion of all SNe Ia. The birthrates in this simulation are lower than those of previous studies, the main reason for which is that new mass-accumulation efficiencies of $\mathrm{H}$-shell flashes are adopted. We also found that the critical mass-accretion rate has significant influence on the birthrates of SNe Ia. Meanwhile, the results of our BPS calculations are sensitive to the values of the common-envelope ejection efficiency.

Key words. binaries: close - stars: evolution - supernovae: general

\section{Introduction}

Type Ia supernovae (SNe Ia) have been successfully used as standard candles for measuring cosmological distances, resulting in the determination of the accelerating expansion of the Universe that is driven by dark energy (e.g., Riess et al. 1998; Perlmutter et al. 1999). There is theoretical consensus that $\mathrm{SNe}$ Ia are thermonuclear explosions of carbon-oxygen white dwarfs (CO WDs; see Hoyle \& Fowler 1960; Nomoto et al. 1997). However, SN Ia progenitor systems and the physics of their explosion mechanism are still uncertain (for recent reviews see Wang \& Han 2012; Hillebrandt et al. 2013; Maoz et al. 2014; Ruiz-Lapuente 2014), which may affect the precision of results of the current cosmological model (e.g., Howell 2011).

Depending on the nature of the mass donor stars, two ways have been proposed by which CO WDs in binary systems can accrete mass towards the Chandrasekhar limit and then cause $\mathrm{SNe}$ Ia. These are the single-degenerate (SD) and the doubledegenerate (DD) model. In the SD model, a CO WD accretes material from a non-degenerate star to increase its mass to the Chandrasekhar mass limit, which then leads to an SN Ia explosion (e.g., Whelan \& Iben 1973; Nomoto et al. 1984). In the DD model, two CO WDs in a close binary are brought together by gravitational wave radiation and merge, which results in an SN Ia explosion (e.g., Webbink 1984; Iben \& Tutukov 1984; Nelemans et al. 2001; Geier et al. 2007; Ruiter et al. 2009;
Chen et al. 2012). For recent observational constraints to theoretical models see, e.g., Maoz et al. (2014), Wang \& Han (2012), Liu et al. (2012a), Wang et al. (2013b) and Graur et al. (2014a,b).

The most commonly considered variation in the SD model is the WD+MS channel (known as the supersoft channel), in which the WD accretes material from a main-sequence (MS) star or a slightly evolved subgiant star (e.g., Hachisu et al. 1996, 1999; Li \& van den Heuvel 1997; Langer et al. 2000; Han \& Podsiadlowski 2004; Wang et al. 2010; Claeys et al. 2014; Ablimit et al. 2014). This channel has been suggested to be accompanied by recurrent novae and supersoft X-ray sources in the past two decades (see Parthasarathy et al. 2007; van den Heuvel et al. 1992; Rappaport et al. 1994). We did not study the SD WD+RG channel here because the SN Ia birthrate from that channel is much lower than the birthrate from the WD+MS channel (e.g., Yungelson \& Livio 1998; Lü et al. 2006; Mennekens et al. 2010; Claeys et al. 2014).

The accretion of H-rich material onto WDs is crucial for understanding SNe Ia from the WD+MS channel. If the massaccretion rate is too high, the WD will evolve into a red-giantlike star as a result of the pileup of the accreted material on its surface. If the mass-accretion rate is too low, the nuclear burning is unstable and $\mathrm{H}$-shell flashes occur, which expels the accreted shell. Thus, there is only a narrow range in which the accreted H-rich material could steadily burn on the surface of 
WDs. This in turn means that theoretical SN Ia birthrates from the WD+MS channel are much lower than those of observations (e.g., Yungelson et al. 1996). An optically thick wind assumption was proposed to solve this problem, in which the H-rich material is transformed into $\mathrm{He}$ at a critical rate, while the unprocessed material is blown off in the form of the optically thick wind (see Hachisu et al. 1996, 1999; Nomoto et al. 2007). However, the optically thick wind assumption is still quite controversial (see Langer et al. 2000). In addition, this assumption does not work when the metallicity is lower than 0.002 (see Kobayashi et al. 1998), while SNe Ia with a metallicity lower than this threshold have been observed (e.g., Prieto et al. 2008; Badenes et al. 2009).

Ma et al. (2013) recently proposed an alternative way to replace the optically thick wind regimes, which is called the superEddington wind scenario. In this scenario, the super-Eddington wind is triggered when the luminosity of the accreting WD exceeds the Eddington luminosity. The super-Eddington wind can naturally prevent the CO WDs with high mass-accretion rates from forming red-giant-like stars. In contrast to the optically thick wind, the super-Eddington wind does not significantly depend on the metallicity, which could be triggered even for $Z=10^{-6}$ (see Fig. 4 of Ma et al. 2013). This indicates that this scenario may contribute to $\mathrm{SNe}$ Ia at high redshifts. In this work, we aim to investigate theoretical SN Ia birthrates and delay-time distributions of the WD+MS channel by adopting the super-Eddington wind scenario.

As mentioned above, at low mass-accretion rates, the accreting WD would experience $\mathrm{H}$-shell flashes similar to nova outbursts. However, H-shell flashes are poorly understood because of the numerical difficulties in modeling nova outbursts. For example, only one cycle of a $\mathrm{H}$-shell flash has been followed in most simulations of nova outbursts; a multicycle evolution of the H-shell flash is difficult to compute (e.g., Kovetz \& Prialnik 1985; Iben et al. 1992; Schwartzman et al. 1994). The H-shell flashes are important for understanding the SN Ia progenitor scenario studied in this paper; a low mass-accumulation efficiency of $\mathrm{H}$-shell flashes will lead to a low birthrate of $\mathrm{SNe}$ Ia. Denissenkov et al. (2013a) recently constructed multicycle nova evolutionary sequences with WDs using the stellar evolution code called modules for experiments in stellar astrophysics (MESA). Adopting a similar method to Denissenkov et al. (2013a), Ma et al. (2013) obtained the mass-accumulation efficiency of $\mathrm{H}$-shell flashes for a wide range of WD masses. A more detailed binary population synthesis (BPS) approach is needed to study the influence of the H-shell flashes on the final results and to give SN Ia birthrates, which is also addressed in this work.

This paper is organized as follows: the numerical code for the binary evolution calculations is described in Sect. 2, and the corresponding results are provided in Sect. 3. The BPS method and results are presented in Sect. 4. Our discussion and summary are then presented in Sect. 5 .

\section{Numerical code for binary evolution}

In the WD+MS channel, the mass donor star is an MS star or a slightly evolved subgiant star that transfers H-rich material onto the surface of the WD. The accreted H-rich material is burned into $\mathrm{He}$, and then the $\mathrm{He}$ is converted into carbon and oxygen, resulting in a mass increase of the WD. We assume that the WD explodes as an SN Ia once its mass grows to $1.378 M_{\odot}$, which is the critical mass limit of nonrotating WDs for carbon

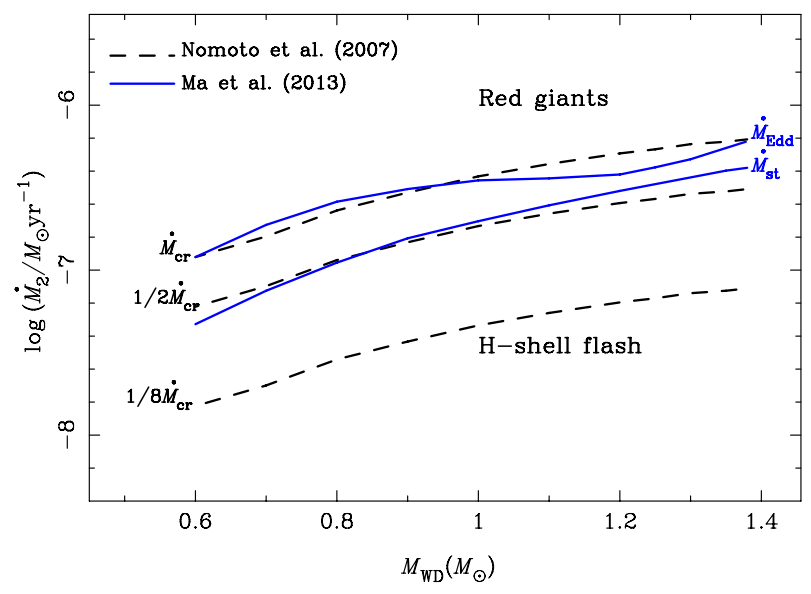

Fig. 1. Properties of H-shell burning on the surface of accreting WDs in the plane of WD mass and mass-accretion rate. We compared the critical mass-accretion rate between the super-Eddington wind scenario and the optically thick wind model. The solid lines are taken from $\mathrm{Ma}$ et al. (2013), while the dashed lines are adopted from Nomoto et al. (2007).

ignition (e.g., Nomoto et al. 1984) ${ }^{1}$. We calculated the evolution of the WD+MS systems using the Eggleton stellar evolution code (Eggleton 1973; Han et al. 1994; Pols et al. 1995, 1998; Eggleton \& Kiseleva-Eggleton 2002). The initial setup and basic input physics for this code are similar to those in Wang et al. (2010). The MS star models in our calculations are composed of metallicity $Z=0.02, \mathrm{H}$ abundance $X=0.70$, and He abundance $Y=0.28$.

The mass transfer begins once the MS star fills its Roche-lobe. We define the values of mass-accumulation efficiency for $\mathrm{H}$-shell burning on the surface of the $\mathrm{WD}, \eta_{\mathrm{H}}$, as

$\eta_{\mathrm{H}}= \begin{cases}\dot{M}_{\mathrm{Edd}} /\left|\dot{M}_{2}\right|, & \left|\dot{M}_{2}\right|>\dot{M}_{\mathrm{Edd}}, \\ 1, & \dot{M}_{\mathrm{Edd}} \geq\left|\dot{M}_{2}\right| \geq \dot{M}_{\mathrm{st}}, \\ \eta_{\mathrm{H}}^{\prime}, & \left|\dot{M}_{2}\right|<\dot{M}_{\mathrm{st}},\end{cases}$

where $\dot{M}_{2}, \dot{M}_{\text {Edd }}$ and $\dot{M}_{\text {st }}$ are the mass-transfer rate, the Eddington critical accretion rate, and the minimum accretion rate for stable $\mathrm{H}$-shell burning, respectively. In the following, we describe in detail: (1) if the mass-transfer rate, $\dot{M}_{2}$, is above the Eddington critical accretion rate, $\dot{M}_{\text {Edd }}$, we assume that the accreted $\mathrm{H}$ steadily burns on the surface of the WD, and that the H-rich material is converted into $\mathrm{He}$ at a rate of $\dot{M}_{\mathrm{Edd}}$,

$$
\begin{aligned}
\dot{M}_{\mathrm{Edd}}= & 5.975 \times 10^{-6}\left(M_{\mathrm{WD}}^{4}-3.496 M_{\mathrm{WD}}^{3}\right. \\
& \left.+4.373 M_{\mathrm{WD}}^{2}-2.226 M_{\mathrm{WD}}+0.406\right),
\end{aligned}
$$

where $M_{\mathrm{WD}}$ is the mass of the WD in units of $M_{\odot}$, and $\dot{M}_{\mathrm{Edd}}$ is in units of $M_{\odot} \mathrm{yr}^{-1}$. The unprocessed material can be lost from the system in the form of the super-Eddington wind with a massloss rate of $\left(\left|\dot{M}_{2}\right|-\dot{M}_{\text {Edd }}\right)$. In Fig. 1, we compare the critical mass-accretion rate of the super-Eddington wind scenario and the optically thick wind model (for a more detailed discussion of this figure see Sect. 5). (2) When $\left|\dot{M}_{2}\right|$ is lower than $\dot{M}_{\text {Edd }}$ but higher than the minimum accretion rate for stable $\mathrm{H}$-shell burning, $\dot{M}_{\text {st }}$, the burning of the $\mathrm{H}$ layer is steady and no mass is lost

1 We did not consider the influence of rotation on the evolution of the accreting WD in this work; the maximum stable mass for a rotating WD may be above the standard Chandrasekhar mass limit (see Uenishi et al. 2003; Yoon et al. 2004; Wang et al. 2014). 


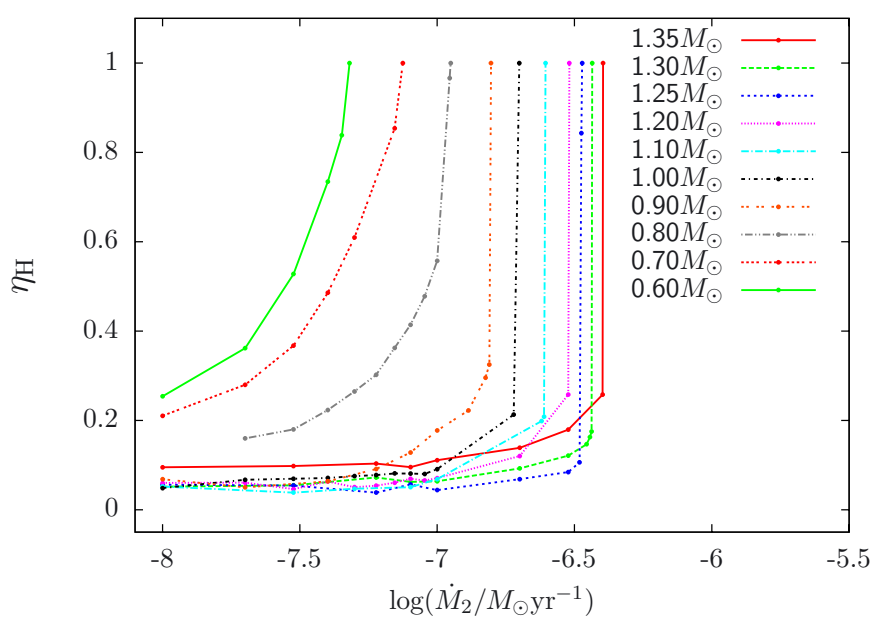

Fig. 2. Mass-accumulation efficiency of H-shell flashes, $\eta_{\mathrm{H}}$, plotted against mass-accretion rate for various initial WD masses. The data points are taken from Ma et al. (2013).

from the system. The values of $\dot{M}_{\mathrm{st}}$ are

$\dot{M}_{\mathrm{st}}=3.057 \times 10^{-7}\left(M_{\mathrm{WD}}^{2}-0.386 M_{\mathrm{WD}}+0.027\right)$,

where $\dot{M}_{\text {st }}$ is in units of $M_{\odot} \mathrm{yr}^{-1}$ (see Fig. 1). (3) H-shell flashes are triggered once $\left|\dot{M}_{2}\right|$ is lower than $\dot{M}_{\text {st }}$. The massaccumulation efficiency for $\mathrm{H}$-shell flashes, $\eta_{\mathrm{H}}^{\prime}$, is linearly interpolated from a detailed grid (see Fig. 2), in which a wide range of WD masses and mass-accretion rate were calculated by Ma et al. (2013).

Helium is assumed to be ignited once the mass of the $\mathrm{He}$ layer under the $\mathrm{H}$-shell burning reaches a certain value. The mass-growth rate of the He layer is defined as

$\dot{M}_{\mathrm{He}}=\eta_{\mathrm{H}}\left|\dot{M}_{2}\right|$.

If He-shell flashes occur, we assume that a part of the layer mass is blown off. In this case, we adopt the prescription of Kato \& Hachisu (2004) for mass-accumulation efficiency of He-shell flashes $^{2}$. We define the mass-growth rate of the WD, $\dot{M}_{\mathrm{CO}}$, as

$\dot{M}_{\mathrm{CO}}=\eta_{\mathrm{He}} \dot{M}_{\mathrm{He}}=\eta_{\mathrm{He}} \eta_{\mathrm{H}}\left|\dot{M}_{2}\right|$,

where $\eta_{\mathrm{He}}$ is the mass-accumulation efficiency of He-shell flashes.

The evolution of the orbital periods of these WD+MS systems is mainly decided by the wind mass-loss of the WD, which carries away the orbital angular momentum of the binaries. We assume that the mass lost from these systems takes away the specific orbital angular momentum of the WD, but the mass lost from the wind of the mass donors is negligible (e.g., Wang et al. 2010). Finally, dense model grids of binary evolution calculations are obtained.

\section{Binary evolution results}

In Fig. 3, we present a representative example of binary evolution calculations. The binary system is in the weak $\mathrm{H}$-shell flash phase at the moment of SN explosion. The initial condition for this binary is $\left(M_{2}^{\mathrm{i}}, M_{\mathrm{WD}}^{\mathrm{i}}, \log \left(P^{\mathrm{i}} /\right.\right.$ day $\left.)\right)=(2.1,0.9,0.4)$, where

\footnotetext{
2 Yoon et al. (2004) argued that the burning of He layer is less violent when rotation is considered, which may increase the massaccumulation efficiency of He-rich material.
}

$M_{2}^{\mathrm{i}}, M_{\mathrm{WD}}^{\mathrm{i}}$ and $P^{\mathrm{i}}$ are the initial masses of the MS star and the CO WD in solar masses, and the initial orbital period in days, respectively. The MS star first fills its Roche-lobe after exhausting the central $\mathrm{H}$ (it now contains a He core), which leads to early Case B mass transfer; the orbital period of the binary system is not changed until the MS star fills its Roche-lobe, see panel b of Fig. 3. The mass-transfer rate $\left|\dot{M}_{2}\right|$ exceeds $\dot{M}_{\text {Edd }}$ soon after the onset of Roche-lobe overflow, resulting in a wind phase in which a part of the transferred mass is blown off in the form of the super-Eddington wind, and the remainder is accumulated on the WD; the wind carries away the specific orbital angular momentum of the WD, which results in the decrease of the orbital period. After about $1.6 \times 10^{6} \mathrm{yr},\left|\dot{M}_{2}\right|$ drops below $\dot{M}_{\text {Edd }}$, but is still higher than $\dot{M}_{\text {st }}$. At this stage, the H-shell burning is stable, but the super-Eddington wind ceases. With the decrease of $\left|\dot{M}_{2}\right|$, the WD quickly enters a weak H-shell flash phase. The orbital period of the binary starts to increase when the accreting WD is more massive than the donor star. The WD always grows in mass until it explodes as an SN Ia in the weak H-shell flash phase. When the WD increases its mass to $1.378 M_{\odot}$, the mass of the donor star is $M_{2}^{\mathrm{SN}}=1.1168 M_{\odot}$ and the orbital period is $\log \left(P^{\mathrm{SN}} /\right.$ day $)=0.2092$.

Figure 4 presents the initial contours for producing $\mathrm{SNe}$ Ia in the orbital period and secondary mass plane for various initial WD masses (i.e., $M_{\mathrm{WD}}^{\mathrm{i}}=0.90,1.0,1.1$ and $1.2 M_{\odot}$ ). The enclosed region almost vanishes for WDs with $0.9 M_{\odot}$, which is then assumed to be the minimum initial WD mass for producing SNe Ia from the WD+MS channel. We note that the critical mass-accretion rate for WDs is still uncertain (e.g., Cassisi et al. 1998; Langer et al. 2000; Nomoto et al. 2007; Shen \& Bildsten 2007; Bours et al. 2013). In the observations, some binaries that contain a neutron star or a black hole can lose their masses at rates that exceed the Eddington accretion rate of the compact object by several times (e.g., Cyg X-2 and SS 433; Podsiadlowski \& Rappaport 2000; Blundell et al. 2001). In Fig. 5, we show the initial contours for producing $\mathrm{SNe}$ Ia after adopting $2 \dot{M}_{\text {Edd }}$ as the critical mass-accretion rate; we did not change the values of the minimum accretion rate for stable $\mathrm{H}$-shell burning (i.e., $\dot{M}_{\text {st }}$ ) in our calculations. This is an unphysical extreme assumption, but we can use this to examine the influence of the critical mass-accretion rate on the final results. From this figure, we can see that the initial contours for producing SNe Ia are significantly enlarged. This is because a larger regime for stable $\mathrm{H}$-shell burning is adopted.

The left boundaries of the initial contours in Figs. 4, 5 are constrained by the condition that Roche-lobe overflow begins when the mass donor star is in the zero-age MS stage, while systems beyond the right boundary experience mass transfer at a relatively high rate as a result of the rapid expansion of the donor stars in the Hertzsprung gap, and they lose too much mass via the super-Eddington wind, preventing the WDs from increasing their masses to the Chandrasekhar mass. The upper boundaries are set by a high mass-transfer rate owing to a high mass ratio between the mass donor star and the WD, while the lower boundaries are constrained by the condition that the mass-transfer rate should be high enough to ensure the mass growth of the WD during $\mathrm{H}$-shell flashes.

\section{BPS assumptions and results}

\subsection{Method of BPS}

To obtain SN Ia birthrates and delay times for the WD+MS channel, we performed a series of Monte Carlo BPS simulations. 

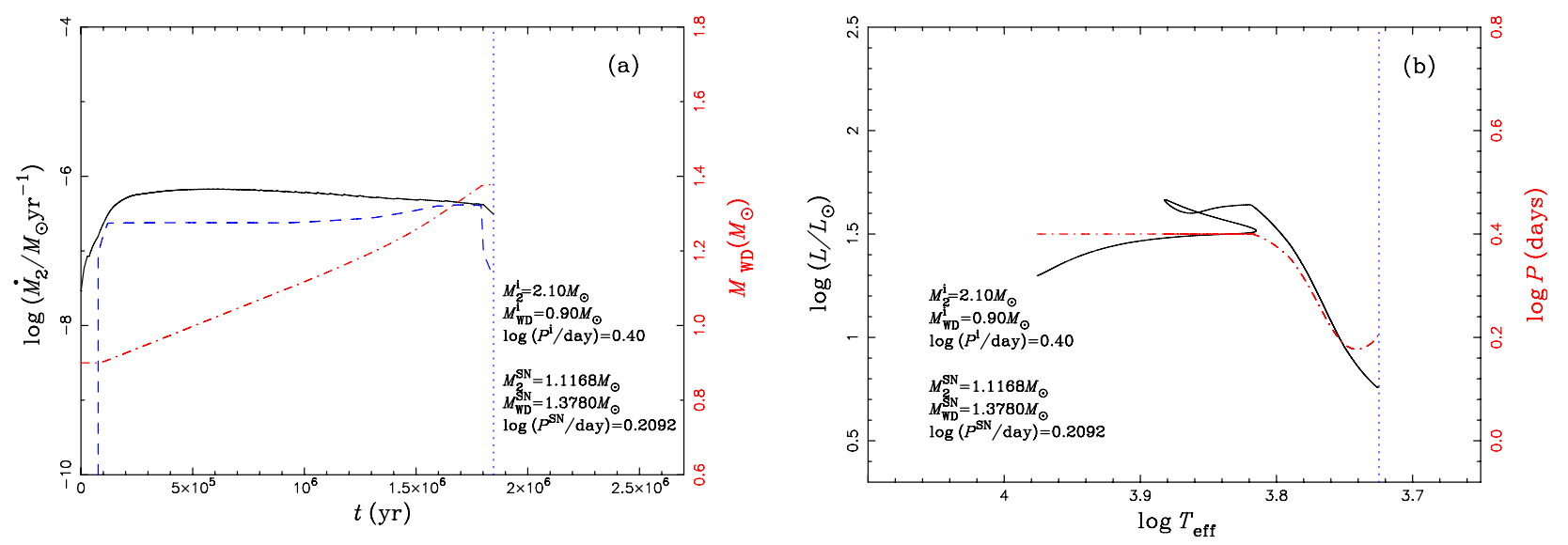

Fig. 3. Representative example of binary evolution calculations, where the binary system is in the weak H-shell flash phase at the moment of the SN explosion. In panel a), the solid, dashed, and dashed-dotted curves present $\dot{M}_{2}, \dot{M}_{\mathrm{CO}}$, and $M_{\mathrm{WD}}$, varying with time. In panel b), the evolutionary track of the donor in the Hertzsprung-Russell diagram is shown as a solid curve, and the evolution of orbital period is shown as a dashed-dotted curve. Dotted vertical lines in both panels indicate the position where the WD produces an SN Ia.

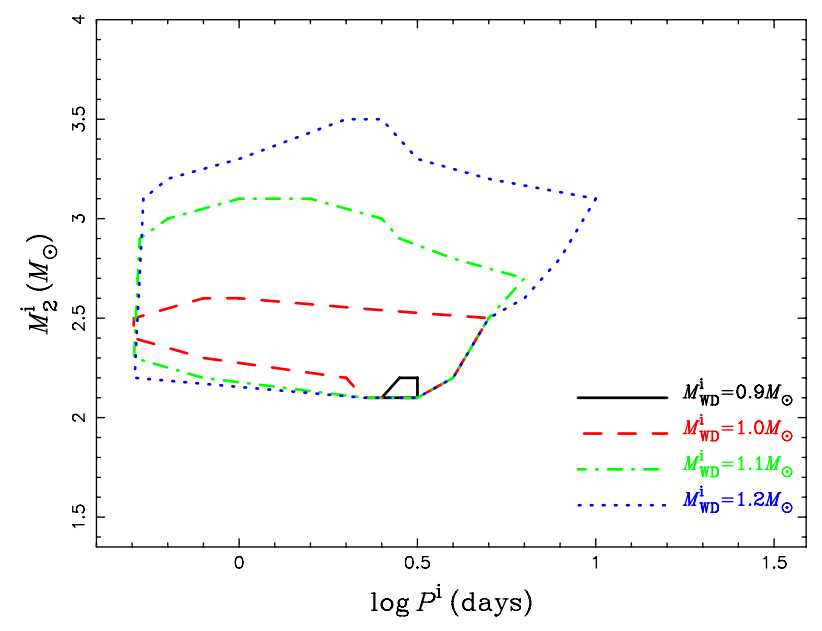

Fig. 4. Regions in the $\log P^{\mathrm{i}}-M_{2}^{\mathrm{i}}$ plane for WD+MS binaries that ultimately produce $\mathrm{SNe}$ Ia for various initial WD masses, where $P^{\mathrm{i}}$ and $M_{2}^{\mathrm{i}}$ are the initial orbital period and the initial mass of the MS star.

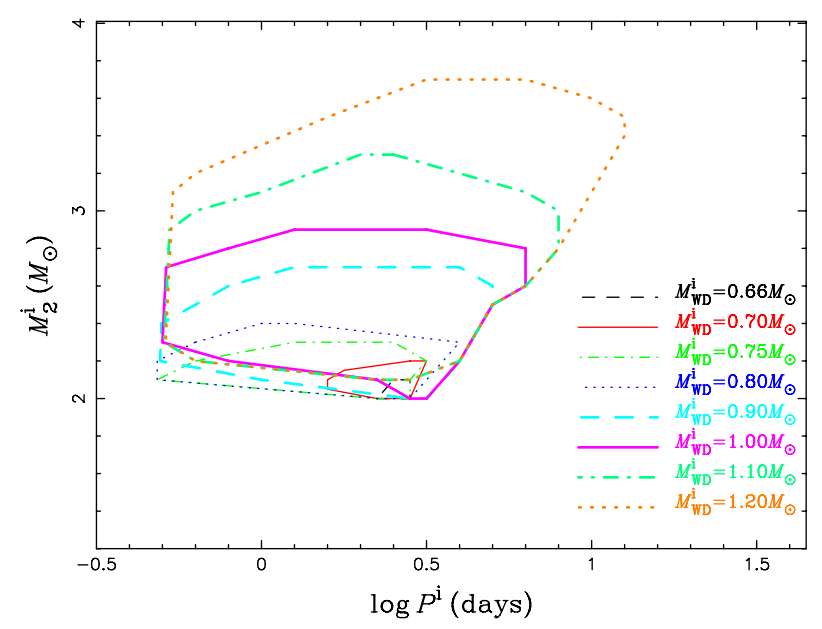

Fig. 5. Similar to Fig. 4 , but for results obtained after adopting $2 \dot{M}_{\text {Edd }}$ as the critical mass-accretion rate.

The random number generator algorithm in our Monte Carlo simulations is adopted from Press et al. (1992). It provides a uniform probability density in the range of $(0,1)$. The repetition period of the random number generator in our simulations is $\gtrsim 10^{18}$, which is infinite for practical calculations.

For each BPS simulation, we employed the rapid binary evolution code of Hurley (Hurley et al. 2002) to simulate the evolution of $10^{7}$ primordial binaries, and adopted a metallicity of $Z=0.02$. These binaries are followed from the star formation to the formation of the WD+MS systems based on three binary evolutionary ways (see Fig. 1 of Wang \& Han 2012). If the initial parameters of a CO WD+MS system at the onset of the Roche-lobe overflow are located in the SN Ia production regions in the plane of $\left(\log P^{\mathrm{i}}, M_{2}^{\mathrm{i}}\right)$ for its specific $M_{\mathrm{WD}}^{\mathrm{i}}$, then an $\mathrm{SN}$ Ia is assumed to occur. We adopted a linear interpolation if $M_{\mathrm{WD}}^{\mathrm{i}}$ is not among the masses listed in Figs. 4, 5.

Seven sets of Monte Carlo BPS simulations were conducted to investigate the birthrates of SNe Ia (see Table 1), in which sets 1 and 2 are the two best models from Camacho et al. (2014). These two best models result in a good overall fit to the properties of the WD+MS binary population in the Sloan Digital Sky Survey (SDSS) Data Release 7. The following basic assumptions for these Monte Carlo simulations were adopted:

(1) A constant star formation rate (SFR) of $5 M_{\odot} \mathrm{yr}^{-1}$ is simply assumed over the past 14 Gyr or, alternatively, it is modeled as a delta function, that is, a single instantaneous starburst $\left(10^{10} M_{\odot}\right.$ in stars is assumed). We intend the constant SFR to approximate spiral galaxies, and the delta function to provide a rough description of elliptical galaxies or globular clusters (e.g., Wang et al. 2013a).

(2) All stars are assumed to be members of binaries. The primordial binary samples are generated via a Monte Carlo method and an initially circular orbit was supposed for all binaries. The distribution of initial orbital separations is assumed to be constant in $\log a$ for wide binaries, where $a$ is the orbital separation (e.g., Han et al. 1995).

(3) The initial mass function (IMF) of the primordial primary star is taken from Miller \& Scalo (1979, MS79). Alternatively, we also considered the IMF of Scalo (1986, S86).

(4) A constant mass-ratio distribution is assumed, that is, $n(q)=$ 1 , or a distribution proportional to the mass ratio, $n(q) \propto q$ (e.g., Camacho et al. 2014).

(5) The common-envelope (CE) evolution is still uncertain (e.g., Zuo \& Li 2014). The standard energy equations are used to describe the output during the CE stage (e.g., 
Table 1. Galactic SN Ia birthrates for seven simulation sets, in which sets 1 and 2 are the two best models from Camacho et al. (2014)

\begin{tabular}{cccccc}
\hline \hline Set & $\alpha_{\mathrm{CE}}$ & $n(q)$ & $\mathrm{IMF}$ & $\dot{M}_{\text {cr }}$ & $v\left(10^{-3} \mathrm{yr}^{-1}\right)$ \\
\hline 1 & 0.3 & 1 & MS79 & $\dot{M}_{\text {Edd }}$ & 0.315 \\
2 & 0.3 & $\propto q$ & MS79 & $\dot{M}_{\text {Edd }}$ & 0.137 \\
3 & 1.0 & 1 & MS79 & $\dot{M}_{\text {Edd }}$ & 0.110 \\
4 & 1.0 & 1 & S 86 & $\dot{M}_{\text {Edd }}$ & 0.070 \\
5 & 1.0 & 1 & MS79 & $2 \dot{M}_{\text {Edd }}$ & 0.955 \\
6 & 3.0 & 1 & MS79 & $\dot{M}_{\text {Edd }}$ & 0.009 \\
7 & 3.0 & 1 & MS79 & $2 \dot{M}_{\text {Edd }}$ & 0.712 \\
\hline
\end{tabular}

Notes. $\alpha_{\mathrm{CE}}=\mathrm{CE}$ ejection efficiency; $n(q)=$ initial mass-ratio distribution; IMF = initial mass function; $\dot{M}_{\mathrm{cr}}=$ critical mass-accretion rate; $v=$ Galactic SN Ia birthrate.

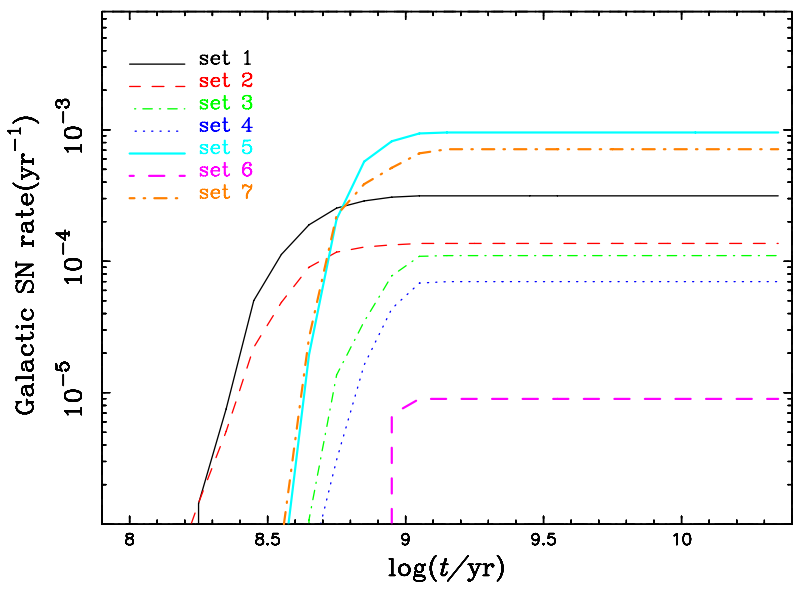

Fig. 6. Evolution of SN Ia birthrates for a constant star-formation rate $\left(S F R=5.0 M_{\odot} \mathrm{yr}^{-1}\right)$ with different BPS simulation sets.

Webbink 1984). There are two uncertain parameters for this prescription of the CE ejection: $\alpha_{\mathrm{CE}}$ (the CE ejection efficiency) and $\lambda$ (a structure parameter that depends on the evolutionary stage of the mass donor). $\lambda$ is usually set to be 0.5 for the purpose of constraining $\alpha_{\mathrm{CE}}$ (see de Kool 1990). Here, we set $\alpha_{\mathrm{CE}}$ to be $0.3,1.0$ and 3.0 to examine its influence on the final results.

\subsection{Results of BPS}

According to the seven sets of simulations for the WD+MS channel, the estimated birthrates of $\mathrm{SNe}$ Ia are strongly sensitive to the choice of some initial conditions, for instance, the critical mass-accretion rate, the $\mathrm{CE}$ ejection efficiency, initial mass-ratio distribution and initial mass function, etc. It is obvious that, if we adopt $2 \dot{M}_{\text {Edd }}$ as the critical mass-accretion rate, the SN Ia birthrate will increase significantly. This is because a larger initial contours for producing SNe Ia are adopted.

In Fig. 6, we show the evolution of SN Ia birthrates with time by adopting metallicity $Z=0.02$ and star formation rate $S F R=5 M_{\odot} \mathrm{yr}^{-1}$. This study presents an SN Ia birthrate of $\sim 0.009-0.315 \times 10^{-3} \mathrm{yr}^{-1}$ by adopting $\dot{M}_{\text {Edd }}$ as the critical accretion rate, which is far lower than the observed value (i.e., $3-4 \times 10^{-3} \mathrm{yr}^{-1}$; Cappellaro \& Turatto 1997) ${ }^{3}$. Thus, we speculate that the WD+MS channel only contributes to a small part

\footnotetext{
3 The upper mass limit for CO WDs has been suggested to be $\sim 1.07 M_{\odot}$ (see Umeda et al. 1999). If this mass limit is adopted in Fig. 4, the SN Ia birthrate from the WD+MS channel will decrease to $\sim 0.009-0.215 \times 10^{-3} \mathrm{yr}^{-1}$.
}

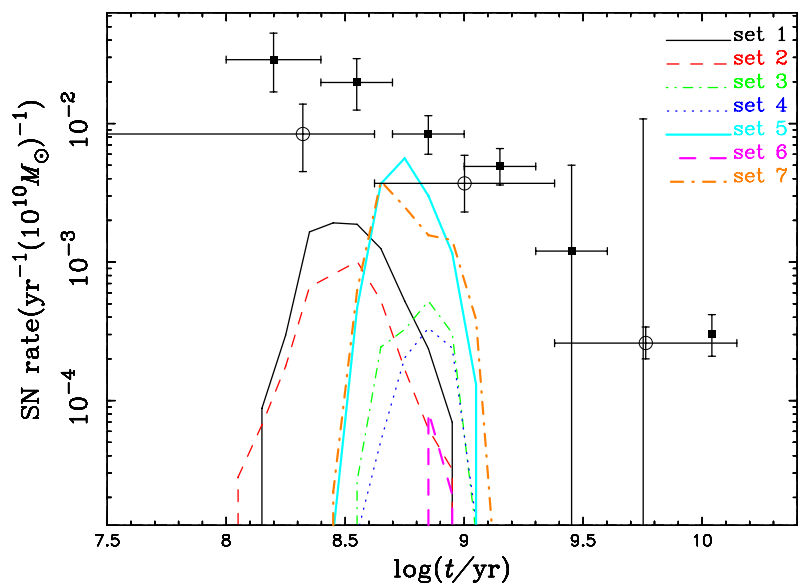

Fig. 7. Delay-time distributions of SNe Ia with different BPS simulation sets. The open circles are taken from Maoz et al. (2011), while the filled squares are adopted from Totani et al. (2008).

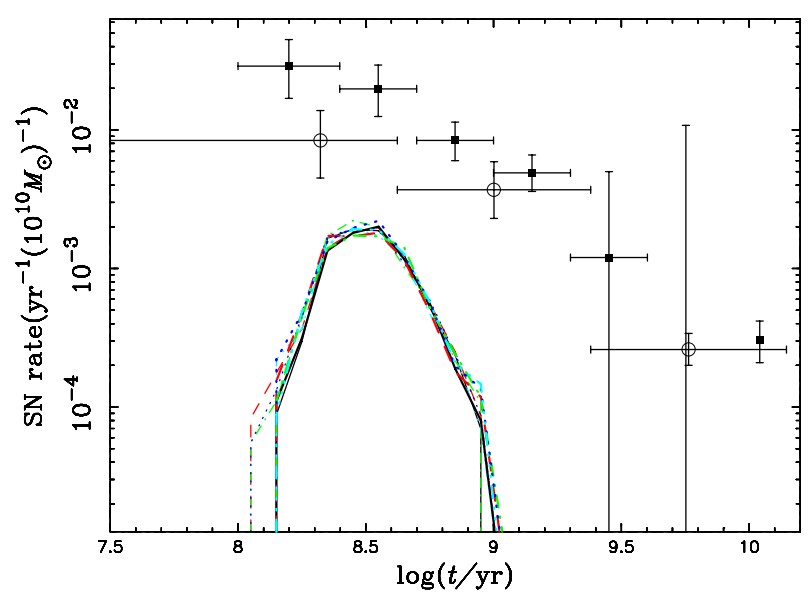

Fig. 8. Similar to Fig. 7, but for the case of set 1, where ten random initial seeds are adopted in our simulations.

of all SNe Ia $(<10 \%)$; some other formation channels or mechanisms may also contribute to $\mathrm{SNe}$ Ia (for a recent review see Wang \& Han 2012). If we adopt $2 \dot{M}_{\text {Edd }}$ as the critical accretion rate, the birthrate of $\mathrm{SNe}$ Ia will increase to $\sim 0.712-0.955 \times$ $10^{-3} \mathrm{yr}^{-1}$ (see sets 5 and 7). This value is still lower than that of observations, but it provides an upper limit on the birthrate of $\mathrm{SNe}$ Ia for the WD+MS channel. This means that the variation of the critical accretion rate has a significant influence on the SN Ia birthrates.

SN Ia delay times are defined as the time interval from star formation to the SN explosion. In Fig. 7, we display the SN Ia delay-time distributions for the WD+MS channel with different BPS simulation sets, which are obtained from a single starburst. From this figure, we can see that the estimated delay times for this channel are $\sim 110 \mathrm{Myr}-1200 \mathrm{Myr}$ after the starburst, which may have a contribution to the SNe Ia with intermediate delay times. Note that a low value of $\alpha_{\mathrm{CE}}$ results in a higher birthrate of SNe Ia. This is because for a low value of $\alpha_{\mathrm{CE}}$ the primordial binary systems need to release more orbital energy to eject the $\mathrm{CE}$ and then produce WD+MS binaries. In this case, WD+MS binaries tend to have slightly closer orbital separations and are more likely to be located in the SN Ia production region (e.g., Figs. 4, 5). We note that, in any Monte Carlo simulations the initial seed of the random number generator results in a given and specific simulated population. In Fig. 8, we show the delay-time 

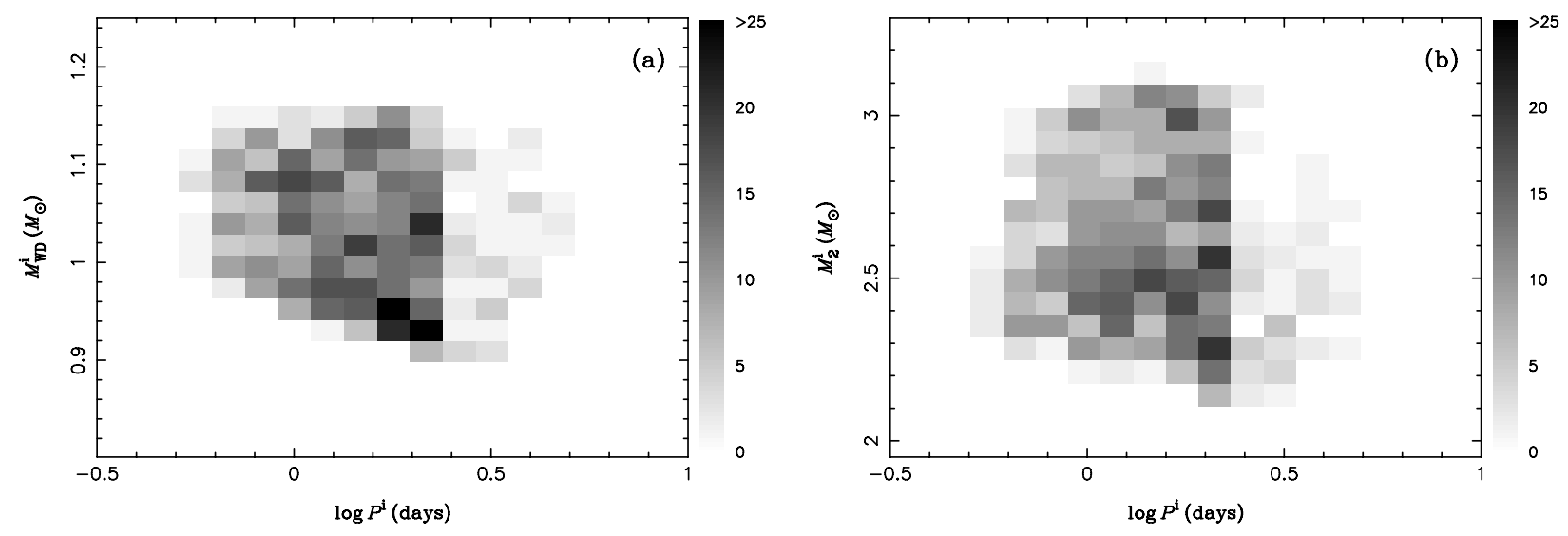

Fig. 9. Distribution of properties of initial WD+MS systems that can ultimately produce SNe Ia. Panel a) shows the results in the log $P^{\mathrm{i}}-M_{\mathrm{WD}}^{\mathrm{i}}$ plane, while panel b) gives the results in the $\log P^{\mathrm{i}}-M_{2}^{\mathrm{i}}$ plane, where $P^{\mathrm{i}}, M_{\mathrm{WD}}^{\mathrm{i}}$ and $M_{2}^{\mathrm{i}}$ are the initial orbital period, the initial WD masses, and the initial mass of the MS star. Here, we present the case of set 1 .

distributions of SNe Ia for the case of set 1, in which ten random initial seeds are adopted in our simulations. From this figure, we found that there is no significant difference among these simulations, meaning that our Monte Carlo BPS results are not sensitive to the initial seed of the random number generator.

In the observations, some WD+MS systems are suggested to be candidates of SN Ia progenitors (for recent reviews see Wang \& Han 2012; Parthasarathy et al. 2007, and references therein). In Fig. 9, we give some properties of initial WD+MS systems that can produce SNe Ia based on our BPS calculations, which would be useful for identifying potential progenitor candidates of SNe Ia. For these WD+MS systems, SN Ia explosions in our simulations occur for the ranges $M_{\mathrm{WD}}^{\mathrm{i}} \sim 0.9-1.2 M_{\odot}$, $M_{2}^{\mathrm{i}} \sim 2.1-3.1 M_{\odot}$ and $P^{\mathrm{i}} \sim 0.5-5$ days. However, recent observations in the SDSS have not found massive MS stars in WD+MS systems, where the MS stars have masses $<0.6 M_{\odot}$ (e.g., Zorotovic et al. 2011; Nebot Gómez-Morán et al. 2011; Rebassa-Mansergas et al. 2012, 2013; Toonen \& Nelemans 2013; Camacho et al. 2014; Li et al. 2014), which cannot afford enough material for the WDs to increase their masses to the Chandrasekhar limit.

\section{Discussion and conclusions}

Previous studies frequently assumed that when the mass-transfer rate is lower than $\frac{1}{2} \dot{M}_{\mathrm{cr}}$ but higher than $\frac{1}{8} \dot{M}_{\mathrm{cr}}$ (see dashed lines in Fig. 1), a weak $\mathrm{H}$-shell flash is triggered, but no mass is lost from the binary system, which would mean that $\mathrm{H}$ is totally converted into He during the mass-accretion phase (e.g., Hachisu et al. 1999; Han \& Podsiadlowski 2004; Meng et al. 2009; Chen \& Li 2009; Wang et al. 2010; Tauris et al. 2013). However, we here adopted a detailed mass-accumulation efficiency for $\mathrm{H}$-shell flashes once the mass-transfer rate is below $\dot{M}_{\text {st }}$ (see Fig. 2), the value of which is lower than in previous studies, leading to a low birthrate of SNe Ia. Note that there is a heated debate about the mass-accumulation efficiency on WDs; it is still uncertain whether nova outbursts remove more mass from the WD than the accreted material (e.g., Prialnik \& Kovetz 1995; Cassisi et al. 1998; Yaron et al. 2005; Idan et al. 2012; Newsham et al. 2014).

Denissenkov et al. (2013b) recently proposed a new type of WDs, the hybrid CONe WDs, which have an unburned CO core surrounded by a thick ONe shell. In a subsequent investigation, Chen et al. (2014) found that this type of hybrid WDs could be as large as $1.3 M_{\odot}$. Following the works of Chen et al. (2014), Meng \& Podsiadlowski (2014) studied the hybrid WD+MS channel of SN Ia progenitors based on a detailed BPS approach and calculated the birthrates $\sim 0.026-0.24 \times 10^{-3} \mathrm{yr}^{-1}$. If this kind of hybrid WDs can really undergo a thermonuclear runaway when increasing their masses to a critical limit, then the SN Ia birthrates from the WD+MS channel can be increased significantly. Note that hybrid WDs could reach a state of explosive carbon ignition, but this depends on some mixing assumptions and the convective Urca process (see Denissenkov et al. 2015).

When the WD in the WD+MS channel explodes as an SN Ia, the mass donor star in this channel would survive and potentially be identifiable (e.g., Liu et al. 2012b; Pan et al. 2014). Tycho G was referred as the surviving companion of Tycho's SN from the WD+MS channel (e.g., Ruiz-Lapuente et al. 2004; Wang \& Han 2010), although it is still debatable (e.g., Ihara et al. 2007; Kerzendorf et al. 2009; Liu et al. 2013). The surviving companions from this channel may also provide a way to explain the formation of single low-mass He WDs in the observations (e.g., Justham et al. 2009; Wang \& Han 2010).

The process of mass accretion onto CO WDs is important for understanding the SD model of SNe Ia, which has a direct effect on the theoretical birthrates of SNe Ia (see also Bours et al. 2013). In this article, by employing the Eggleton stellar evolution code and adopting detailed mass-accumulation efficiencies of $\mathrm{H}$-shell flashes on the WDs, we performed binary evolution calculations for the WD+MS channel in detail based on the super-Eddington wind scenario and computed the initial parameter spaces for producing SNe Ia with various initial WD masses. We found that a CO WD with a mass as low as $0.9 M_{\odot}$ can potentially accrete enough mass and then reach the Chandrasekhar mass limit if we adopt $\dot{M}_{\text {Edd }}$ as the critical accretion rate. By incorporating the results of the detailed binary evolution calculations into a detailed BPS approach, we obtained the evolution of SN Ia birthrates with time. The SN Ia birthrate from the WD+MS channel is much lower than the value obtained from observations. This means that the contributions of the WD+MS channel to the total SN Ia birthrates are quite inefficient. However, this result is strongly sensitive to uncertainties in some initial conditions, especially the efficiency of mass accretion. If we adopt $2 \dot{M}_{\text {Edd }}$ as the critical accretion rate, the SN Ia birthrate is increased significantly. Additionally, the values of $\alpha_{\mathrm{CE}}$ also have an influence on the birthrates of SNe Ia. To set further constraints on the WD+MS channel, large samples of 
observed massive WD+MS systems and surviving companions are needed, and the process of mass accretion on WDs should be examined carefully.

Acknowledgements. We acknowledge the anonymous referee for valuable comments that helped us to improve the paper. We thank Xiangdong Li, Fenghui Zhang and Xiangcun Meng for their useful discussions. We also thank Yan Gao for his kind help to improve the language of this paper. This study is supported by the National Basic Research Program of China (973 program, 2014CB845700), the National Natural Science Foundation of China (Nos. 11322327, 11390374 and U1331117), the Chinese Academy of Sciences (No. XDB09010202), and the Natural Science Foundation of Yunnan Province (Nos. 2013FB083 and 2013HB097).

\section{References}

Ablimit, I., Xu, X.-J., \& Li, X.-D. 2014, ApJ, 780, 80

Badenes, C., Harris, J., Zaritsky, D., \& Prieto, J. L. 2009, ApJ, 700, 727

Blundell, K. M., Mioduszewski, A. J., Muxlow, T. W., Podsiadlowski, P., \& Rupen, M. P. 2001, ApJ, 562, L79

Bours, M. C. P., Toonen, S., \& Nelemans, G. 2013, A\&A, 552, A24

Camacho, J., Torres, S., García-Berro, E., et al. 2014, A\&A, 566, A86

Cappellaro, E., \& Turatto, M. 1997, in Thermonuclear Supernovae, eds.

P. Ruiz-Lapuente, R. Cannal, \& J. Isern (Dordrecht: Kluwer), 77

Cassisi, S., Iben, I., \& Tornambè, A. 1998, ApJ, 496, 376

Chen, W.-C., \& Li, X.-D. 2009, ApJ, 702, 686

Chen, X., Jeffery, C. S., Zhang, X., \& Han, Z. 2012, ApJ, 755, L9

Chen, M. C., Herwig, F., Denissenkov, P. A., \& Paxton, B. 2014, MNRAS, 440, 1274

Claeys, J. S. W., Pols, O. R., Izzard, R. G., Vink, J., \& Verbunt, F. W. M. 2014, A\&A, 563, A83

de Kool, M. 1990, ApJ, 358, 189

Denissenkov, P. A., Herwig, F., Bildsten, L., \& Paxton, B. 2013a, ApJ, 762, 8

Denissenkov, P. A., Herwig, F., Truran, J. W., \& Paxton, B. 2013b, ApJ, 772, 37

Denissenkov, P. A., Truran, J. W., Herwig, F., et al. 2015, MNRAS, 447, 2696

Eggleton, P. P. 1973, MNRAS, 163, 279

Eggleton, P. P., \& Kiseleva-Eggleton, L. 2002, ApJ, 575, 461

Geier, S., Nesslinger, S., Heber, U., et al. 2007, A\&A, 464, 299

Graur, O., Maoz, D., \& Shara, M. M. 2014a, MNRAS, 442, L28

Graur, O., Rodney, S. A., Maoz, D., et al. 2014b, ApJ, 783, 28

Hachisu, I., Kato, M., \& Nomoto, K. 1996, ApJ, 470, L97

Hachisu, I., Kato, M., Nomoto, K., \& Umeda, H. 1999, ApJ, 519, 314

Han, Z., \& Podsiadlowski, P. 2004, MNRAS, 350, 1301

Han, Z., Podsiadlowski, Ph., \& Eggleton, P. P. 1994, MNRAS, 270, 121

Han, Z., Podsiadlowski, Ph., \& Eggleton, P. P. 1995, MNRAS, 272, 800

Hillebrandt, W., Kromer, M., Röpke, F. K., \& Ruiter, A. J. 2013, Frontiers of Physics, 8, 116

Howell, D. A. 2011, Nature Comm., 2, 350

Hoyle, F., \& Fowler, W. A. 1960, ApJ, 132, 565

Hurley, J. R., Tout, C. A., \& Pols, O. R. 2002, MNRAS, 329, 897

Iben, I., \& Tutukov, A. V. 1984, ApJS, 54, 335

Iben, I., Fujimoto, M. Y., \& MacDonald, J. 1992, ApJ, 388, 521

Idan, I., Shaviv, N. J., \& Shaviv, G. 2012, J. Phys. Conf. Ser., 337, 012051

Ihara, Y., Ozaki, J., Doi, M., et al. 2007, PASJ, 59, 811

Justham, S., Wolf, C., Podsiadlowski, P., \& Han, Z. 2009, A\&A, 493, 1081

Kato, M., \& Hachisu, I. 2004, ApJ, 613, L129

Kerzendorf, W. E., Schmidt, B. P., Asplund, M., et al. 2009, ApJ, 701, 1665

Kobayashi, C., Tsujimoto, T., Nomoto, K., Hachisu, I., \& Kato, M. 1998, ApJ, 503, L155

Kovetz, A., \& Prialnik, D. 1985, ApJ, 291, 812

Langer, N., Deutschmann, A., Wellstein, S., \& Höflich, P. 2000, A\&A, 362, 1046 Li, X.-D., \& van den Heuvel, E. P. J. 1997, A\&A, 322, L9

Li, L.-F., Zhang, F., Han, Q., Kong, X., \& Gong, X. 2014, MNRAS, 445, 1331

Liu, J.-F., Di Stefano, R., Wang, T., \& Moe, M. 2012a, ApJ, 749, 141
Liu, Z.-W., Pakmor, R., Röpke, F. K., et al. 2012b, A\&A, 548, A2 Liu, Z.-W., Pakmor, R., Röpke, F. K., et al. 2013, A\&A, 554, A109 Lü, G., Yungelson, L., \& Han, Z. 2006, MNRAS, 372, 1389

Ma, X., Chen, X., Chen, H., Denissenkov, P. A., \& Han, Z. 2013, ApJ, 778, L32

Maoz, D., Mannucci, F., Li, W., et al. 2011, MNRAS, 412, 1508

Maoz, D., Mannucci, F., \& Nelemans, G. 2014, ARA\&A, 52, 107

Meng, X., \& Podsiadlowski, Ph. 2014, ApJ, 789, L45

Meng, X., Chen, X., \& Han, Z. 2009, MNRAS, 395, 2103

Mennekens, N., Vanbeveren, D., De Greve, J. P., \& De Donder, E. 2010, A\&A, $515, \mathrm{~A} 89$

Miller, G. E., \& Scalo, J. M. 1979, ApJS, 41, 513 (MS79)

Nebot Gómez-Morán, A., Gänsicke, B. T., Schreiber, M. R., et al. 2011, A\&A, 536, A43

Nelemans, G., Yungelson, L. R., Portegies Zwart, S. F., \& Verbunt, F. 2001, A\&A, 365, 491

Newsham, G., Starrfield, S., \& Timmes, F. 2014, ASP Conf. Ser., 490, 287

Nomoto, K., Thielemann, F.-K., \& Yokoi, K. 1984, ApJ, 286, 644

Nomoto, K., Iwamoto, K., \& Kishimoto, N. 1997, Science, 276, 1378

Nomoto, K., Saio, H., Kato, M., \& Hachisu, I. 2007, ApJ, 663, 1269

Pan, K.-C., Ricker, P. M., \& Taam, R. E. 2014, ApJ, 792, 71

Parthasarathy, M., Branch, D., Jeffery, D. J., \& Baron, E. 2007, New Astron. Rev., 51, 524

Perlmutter, S., Aldering, G., Goldhaber, G., et al. 1999, ApJ, 517, 565

Podsiadlowski, P., \& Rappaport, S. 2000, ApJ, 529, 946

Pols, O. R., Tout, C. A., Eggleton, P. P., \& Han, Z. 1995, MNRAS, 274, 964

Pols, O. R., Schröder, K. P., Hurly, J. R., Tout, C. A., \& Eggleton, P. P. 1998, MNRAS, 298, 525

Press, W. H., Teukolsky, S. A., Vetterling, W. T., \& Flannery, B. P. 1992, in Numerical recipes in FORTRAN. The art of scientific computing (Cambridge University Press), 271

Prialnik, D., \& Kovetz, A. 1995, ApJ, 445, 789

Prieto, J. L., Stanek, K. Z., \& Beacom, J. F. 2008, ApJ, 673, 999

Rappaport, S., Di Stefano, R., \& Smith, J. D. 1994, ApJ, 426, 692

Rebassa-Mansergas, A., Nebot Gómez-Morán, A., Schreiber, M. R., et al. 2012, MNRAS, 419, 806

Rebassa-Mansergas, A., Agurto-Gangas, C., Schreiber, M. R., Gänsicke, B. T., \& Koester, D. 2013, MNRAS, 433, 3398

Riess, A., Filippenko, A. V., Challis, P., et al. 1998, AJ, 116, 1009

Ruiter, A. J., Belczynski, K., \& Fryer, C. L. 2009, ApJ, 699, 2026

Ruiz-Lapuente, P. 2014, New Astron. Rev., 62, 15

Ruiz-Lapuente, P., Comeron, F., Méndez, J., et al. 2004, Nature, 431, 1069

Scalo, J. M. 1986, Fund. Cosm. Phys., 11, 1 (S86)

Schwartzman, E., Kovetz, A., \& Prialnik, D. 1994, MNRAS, 269, 323

Shen, K. J., \& Bildsten, L. 2007, ApJ, 660, 1444

Tauris, T. M., Sanyal, D., Yoon, S.-C., \& Langer, N. 2013, A\&A, 558, A39

Toonen, S., \& Nelemans, G. 2013, A\&A, 557, A87

Totani, T., Morokuma, T., Oda, T., Doi, M., \& Yasuda, N. 2008, PASJ, 60, 1327

Uenishi, T., Nomoto, K., \& Hachisu, I. 2003, ApJ, 595, 1094

Umeda, H., Nomoto, K., Yamaoka, H., \& Wanajo, S. 1999, ApJ, 513, 861

van den Heuvel, E. P. J., Bhattacharya, D., Nomoto, K., \& Rappaport, S. A. 1992, A\&A, 262, 97

Wang, B., \& Han, Z. 2010, MNRAS, 404, L84

Wang, B., \& Han, Z. 2012, New Astron. Rev., 56, 122

Wang, B., Li, X.-D., \& Han, Z. 2010, MNRAS, 401, 2729

Wang, B., Justham, S., \& Han, Z. 2013a, A\&A, 559, A94

Wang, X.-F., Wang, L., Filippenko, A. V., Zhang, T., \& Zhao, X. 2013b, Science, 340,170

Wang, B., Justham, S., Liu, Z., et al. 2014, MNRAS, 445, 2340

Webbink, R. F. 1984, ApJ, 277, 355

Whelan, J., \& Iben, I. 1973, ApJ, 186, 1007

Yaron, O., Prialnik, D., Shara, M. M., \& Kovetz, A. 2005, ApJ, 623, 398

Yoon, S.-C., Langer, N., \& Scheithauer, S. 2004, A\&A, 425, 217

Yungelson, L., \& Livio, M. 1998, ApJ, 497, 168

Yungelson, L., Livio, M., Truran, J. W., Tutukov, A., \& Fedorova, A. 1996, ApJ, 466, 890

Zorotovic, M., Schreiber, M. R., \& Gänsicke, B. T. 2011, A\&A, 536, A42

Zuo, Z.-Y., \& Li, X.-D. 2014, MNRAS, 442, 1980 